\title{
Medical Imaging System and the Health Care Delivery System in Nigeria: An Overview
}

\author{
Oladele Stephen Adeola \\ Department of Computer Science, Federal University of Technology Akure, Akure, Nigeria
}

\section{Email address:}

osadeola@futa.edu.ng, deleaeola@yahoo.com

\section{To cite this article:}

Oladele Stephen Adeola. Medical Imaging System and the Health Care Delivery System in Nigeria: An Overview. International Journal of Medical Imaging. Vol. 9, No. 1, 2021, pp. 45-56. doi: 10.11648/j.ijmi.20210901.15

Received: December 11, 2020; Accepted: January 4, 2021; Published: January 30, 2021

\begin{abstract}
The concept of using an imaging system to effectively diagnose and treat patients is well embraced in medical health care delivery systems of most advanced countries of the world. This has brought a lot of efficiency, availability and a high degree of leverage to the systems. But implementing such a system in third world countries especially a state such as Nigeria is still a challenge. The prospect of implementation, however, is enormous. This study aims at providing first-hand information about medical imaging to the health care practitioners. It is also intended to give a brief overview of the state of medical imaging in the health care delivery system with a focus on Nigeria. It additionally gives an insight into its challenges and discusses the benefits and prospects of the computer imaging system in the system. A methodological review of the current state of medical imaging system was examined with particular focus on its relationship to the health care delivery system in Nigeria. The use, benefits, method of generating as well as challenges and the huge potential of medical imaging in the advancement of the health care delivery system in Nigeria were examined. The study exposes the latest trend in medical imaging technologies and the relevancies to the Nigeria health care delivery system. Also, it examines the use of imaging technologies to treat various medical conditions of a patient in pre-medical and post-medical therapy. Also, up-to-date information about medical imaging focusing on Nigeria health care delivery system was discussed. It provides the first-hand informative source for medical practitioners as well as the general public when evaluating the need and importance of medical images to the overall health care delivery system in Nigeria. It reveals the uses, process in generating, capturing, storage and reporting of medical imaging as well as exploring the challenges and benefit of an imaging system in advancing health care delivery system in Nigeria. The study exposes the latest trend in medical imaging technologies and the relevancies to the Nigeria health care delivery system.
\end{abstract}

Keywords: Imaging Technology, Medical Imaging, Health Care, Medical Information, Information Technology, Network, Radiological Information System

\section{Introduction}

Health is the wealth of a nation. The health of the citizenry is directly or indirectly related to how productive a nation would be in term of manpower and issues relating to creating wealth. Also, the health can be directly related to the level of medical infrastructure development and how the infrastructure is harnessed to bridge the gap between the rich and the poor and how it is used to raise the level of productivity. This is most imperative for any meaningful development goal(s) to be achieved in the so call third-world countries and developing nations. Nigeria, as a vastly developing economy, needs to harness all resources at her disposal to improve the health of its citizenries for the sustenance of any meaningful economic and sociodevelopment goals. One of the areas to focus on is the development of a solid medical imaging system for her health care delivery system. Medical imaging is highly essential to provide more accurate diagnoses for medical treatment and therapy. The advancement in the research outcome of big data has created ample opportunities for improving medical care. Big data analysis has the potential of providing real-time imaging diagnostic system in the medical health care delivery system. This would improve coverage 
time and efficiency of treatment.

Furthermore, the majority of government hospitals in Nigeria are devoid of modern imaging techniques and appliances for the modern health care delivery system. Many government-owned hospitals with ageing imaging system place less emphasis on acquiring modern system and the physicians there prefer to refer patients to a privately owned hospital where they have a personal interest. Even a simple ultrasound system is not functional in many government hospitals in Nigeria. Patients are, on many occasions, refer to a private clinic or government teaching hospital to access modern imaging systems. Those systems range from Ambulatory Surgery Centres (ASCs), Radiology Centers, Hospitals and Emergency Care Centers and the applications could vary from Chronic Disorders, Bone Tumors, Acute Injuries and other applications.

The discussion in this paper focuses on medical imaging in Nigeria health care delivery system, it challenges, prospect and what needs to be done to transform the system.

\section{Background Studies}

Health care delivery system in developing countries is very poor needing more to be desired. Nigeria, with a population of over 178.5 million and geographical area of about 923,768 sqkm [1], has most of its citizens living in rural areas where road, electricity and essentials of life are missing. The infant mortality rate is high and, in some rural areas, the death is often attributed to witches, wizard and the children belonging to "Ogbannjes" by the local inhabitants. The medical history of the patient is virtually missing. Most rural settings have no hospital, no premedical history of patients not to talk of digitalized imaging for fast and proper diagnosis. Most of the communities practices non-orthodox medicine. Though some of these traditional medicines are effective but the fact that the therapy often lacks dosage administration and usually ignore the medical history of the patient account for the majority of the death associated with the traditional practices. The trend is changing; the government is setting up tradomedical agencies for effective coordination and integration of traditional medicine into the mainstream orthodox medicine. Research institutions are gradually embracing research in some traditional medicine found to be effective in disease treatment and management. Traditional healers and medical practitioners need to be enlightened about the hazard in improper dosage administration of trado-medicine, ignoring standard medical record, lack of knowledge to interpret medical imaging as well as lack of awareness in modern practices in medical therapies [2]. Compounding the problems, medical imaging is excluded from medical therapy in the majority of the therapies in Nigeria. This would have compensated for the lack of proper medical history.

Digital medical imaging involves the use of various digital and analogue techniques use in capturing, storing, transmission, display, analysis, visualization and reporting of medical imaging of the patient. It typically encompasses several linked processes each affected by different technologies. Radiologists and medical doctors both use medical imaging to advance patient treatment and therapy. Whereas the radiographers use the technologies to diagnose and treat diseases, the doctors use the technologies to test the efficacy of certain therapy they have adopted in treating patients. Imaging technologies have evolved over the years and there are many types of medical imaging, with more methods for imaging being invented as technology advances. radiography, magnetic resonance imaging (MRI), nuclear medicine, ultrasound, et cetera are notably used in modern medicine.

Ojoma and Olayemi lamented the absence of radiotherapy centres for prevention and treatment of cancer in Nigeria and emphasize the importance of screening for a preventive purpose which, according to him, the equipment is not even present in the country [3]. For example, mammography and linear accelerators are used for cancer treatment but unfortunately, the equipment is not available in most of the health centres in Nigeria. In the United Kingdom, there is a screening protocol whereby every woman conducts screening at the age of 40 whether you have a lump or not. Also, radiography skill is not at its best in Nigeria.

In this work, medical imaging would be reviewed to unlock the preliminary knowledge in the field to the prospective medical imaging researcher and the medical care practitioners. Also, challenges and the prospect of medical imaging as related to Nigeria shall be discussed. Finally, a way forward for the integration of medical imaging into our health care delivery system would be examined.

\section{Scope of the Study}

The study attempts to discuss some topical medical imaging system in use in modern health care delivery system with an emphasis on the Nigeria health sector and limit itself to known imaging devices system popularly use in Nigeria and beyond. The study does not assume that the discussed imaging systems are exhaustive.

\section{Literature Review}

Medical imaging is used for diagnostic purposes. The inner part of body play host to the majority of medical issues that occur in the body and it becomes imperative that any technology that paves way for viewing the source and diagnoses the problem in a noninvasive way becomes extremely useful. This makes the challenge of diagnosing medical issues easier and more accurate. Medical imaging creates pictures of tissues, bones and organs inside the human body system and these technologies assist doctors to diagnose and assess diseases in medical therapy. The technology is described as the technique of producing visual representations of areas inside the human body to diagnose problems and monitor treatment [4]. In practice, the technologies are based on x-ray which uses radiation to create images, Computed tomography (CT or CAT scan) which combines $\mathrm{x}$-rays and a computer to create 360 -degree 
pictures of the spine and internal organs. The images in CTs are more detailed than traditional x-rays. Others are Ultrasound which uses high-frequency sound waves to create images, Magnetic resonance imaging (MRI) which uses a strong magnetic field and radio waves to create images of the body that cannot be seen clearly with x-rays, and finally, Nuclear medicine makes it possible for early detection of cancer and other serious illnesses. For this review, the technology would just be classified as X-rays based and non$\mathrm{X}$-rays based technologies.

\subsection{X-rays Based Technologies}

\section{i. Conventional Radiography}

Conventional radiography involves the use of electromagnetic radiation to examine the inner body part by taking its images. Popular among the radiography is the $\mathrm{x}$-ray imaging. This typically involves an x-ray machine beaming high-energy waves onto the body. Whereas the hard tissues such as bones absorb the waves, the soft tissues like skin and organs do not, the result of the x-ray is transferred on a thin film thereby exposing the body parts that absorb the waves in white leaving the unabsorbed materials in black. The image is shown in Figure 1 uses a KUB radiography diagnostic tool that uses $\mathrm{x}$-ray imaging to view the kidneys, ureters, and bladder to evaluate the condition of these organs, and potential urinary health conditions. Figure 1 uses a typical $x-$ ray of the human body part [5].

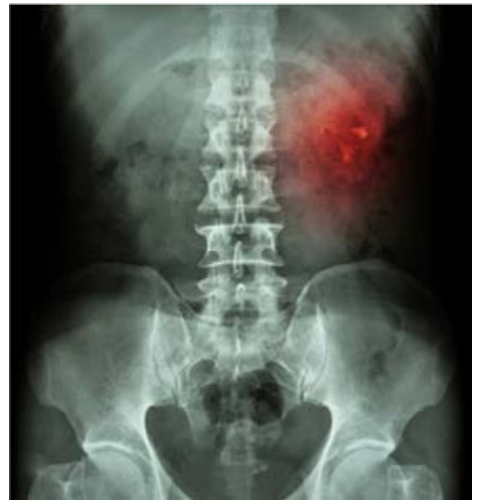

Figure 1. X-ray Application in Medicine [5].

\section{ii. Intravenous Urography}

Intravenous Urography (IVU), also known as intravenous pyelography (IVP) or excretory urography (EU),) is a test that uses X-rays and a special dye to help assess your kidneys, ureters, bladder and urethra. It is used to assess problems in kidneys, ureters, bladder and urethra. These structures make up the urinary tract. This exam has been largely replaced by CT urography. Radiology.org describes it as an x-ray examination of the kidneys, ureters and urinary bladder that uses iodinated contrast material injected into veins [6]. Figure 2 shows the radiography procedure being carried out on a patient. Also, Figure 3(a) and Figure 3(b) depict male patient with right-sided flank pain and right ureter was dilated with the cause not obvious but the Urine examination revealed tuberculosis.

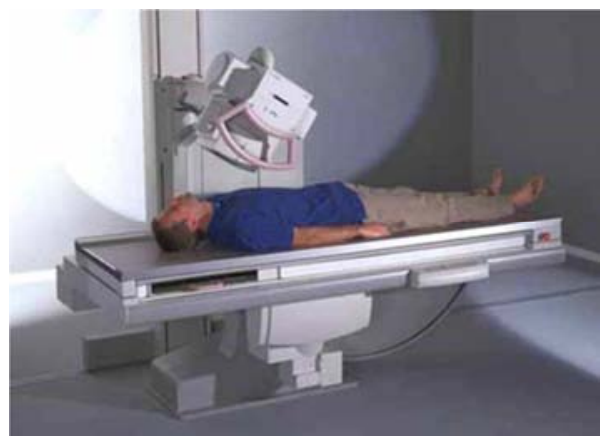

Figure 2. Procedure in Radiography [6].

Intravenous urography is indicated for the following body conditions:

1. check for the normal function of kidneys.

2. check for anatomical variants or congenital anomalies (e.g. horse-shoe kidney).

3 . check the course of the ureters.

4. detect and localize a ureteric obstruction (urolithiasis).

5. assess for synchronous upper tract disease in those with bladder transitional cell carcinoma (TCC). [6]

Thus, with this method, it is possible to diagnose symptoms such as blood in the urine or pain in the side or lower back. The IVP exam can also enable the radiologist to detect problems within the urinary tract resulting from kidney stones, congenital anomalies of the urinary tract, scarring from urinary tract infection, enlarged prostate, surgery on the urinary tract, tumours in the kidney, ureters or urinary bladder. Figure $3 \mathrm{a}$ and $3 \mathrm{~b}$ respectively male patient with right-sided flank pain and the right ureter was dilated, but the cause was not obvious. Also, in Figure 3a, no radiopaque calculus was seen and in Figure 3b, no vesicoureteral reflux was seen on colour Doppler. Also, urine examination revealed tuberculosis

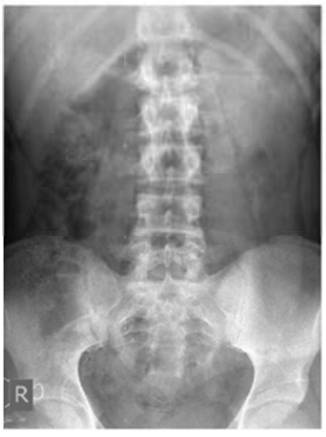

(a)

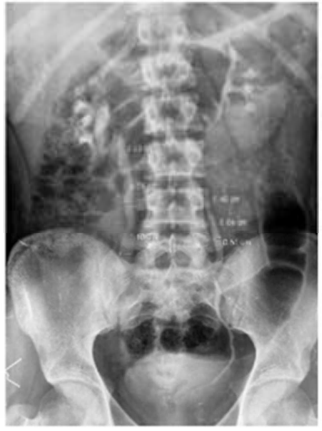

(b)
Figure 3. (a) Male patient with right-sided flank pain (b) IVU: right ureter was dilated, but the cause was not obvious. [7].

\section{iii. Microbeam Radiation Therapy (MRT) for Inoperable Cancer}

Active research on an innovative radiation treatment led by the University of Saskatchewan (USask) researchers at the Canadian Light Source (CLS) which could supplement existing methods of conventional radiation therapy for inoperable brain and spinal tumours. This approach was documented based on the method of Microbeam Radiation 
Therapy (MRT) uses very high dose, synchrotron-generated X-ray beams - narrower than a human hair - to blast tumours with radiation while sparing healthy tissue [8]. Doing this allows the MRT to deliver an additional dose of radiation to a tumour after maximum conventional radiation therapy has been tried and thus providing patients with another treatment that could extend their lives.

\section{iv. Fluoroscopy}

Fluoroscopy is an imaging technique used to obtain realtime images of the internal structures of a patient through the use of a fluoroscope. It involves the use of photo-electronic components that shows a continuous X-ray image on a monitor, much like an X-ray movie. The X-ray beam is passed through the body and the image is transmitted to a monitor so the movement of a body part or an instrument or contrast agent ("X-ray dye") through the body can be seen in detail. The following ranges of varieties of examinations and procedures in which fluoroscopy is used to diagnose or treat patients:

1. Barium X-rays and enemas (to view the gastrointestinal tract).

2. Catheter insertion and manipulation (to direct the movement of a catheter through blood vessels, bile ducts or the urinary system).

3. Placement of devices within the body, such as stents (to open narrowed or blocked blood vessels).

4. Angiograms (to visualize blood vessels and organs).

5. Orthopaedic surgery (to guide joint replacements and treatment of fractures). [9]

Fluoroscopy is not without risk just like a conventional Xray procedure. The radiation dose absolved by the body as a result of fluoroscopy can result in relatively high radiation doses especially when administered for a long period.

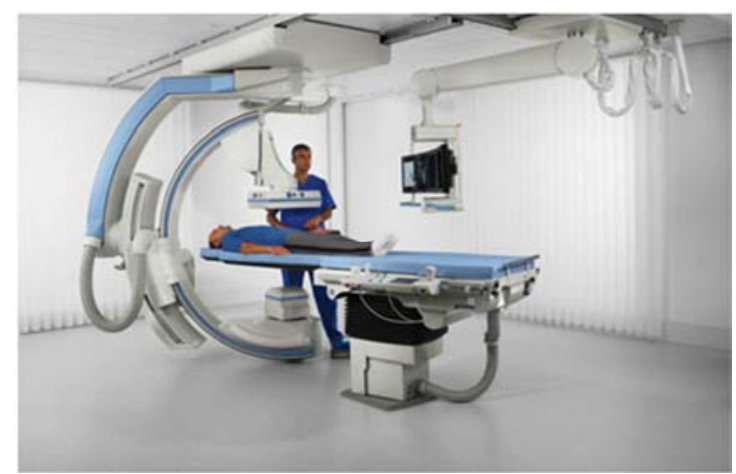

Figure 4. Image Courtesy of Siemens Healthcare USA (culled from U.S. Food and Drug Administration [9].

Radiation-related risks associated with fluoroscopy according to the U.S. Food and Drug Administration include: radiation-induced injuries to the skin and underlying tissues ("burns"), which occur shortly after the exposure, and radiation-induced cancers, which may occur sometime later in life.

Statistically, these effects are very small. Figure 4 shows a patient undergoing the fluoroscopy treatment while figure 5 shows fluoroscopy being used for real-time X-ray to image the internal organs of a patient.

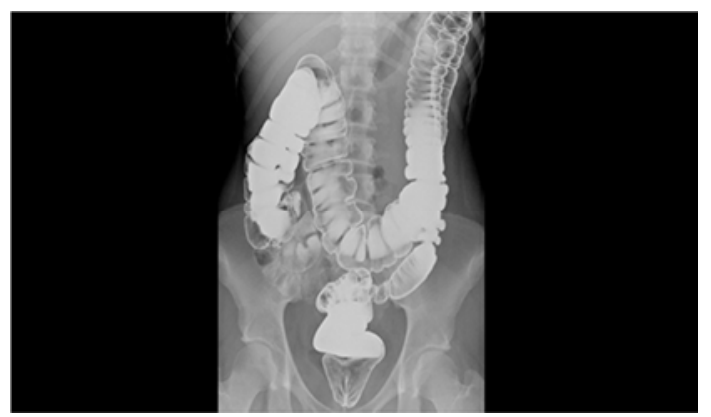

Figure 5. Fluoroscopy Sample of internal organs (courtesy of Radiology Associates).

\section{v. Positron Emission Tomography (PET)}

A Positron Emission Tomography (PET) scan is an imaging test that shows how tissues and organs of the body are functioning. It is a nuclear medicine procedure that measures the metabolic activity of the cells of body tissues. PET is a combination of nuclear medicine and biochemical analysis. It is mostly employed in patients with brain or heart conditions and cancer [10]. PET visualizes the biochemical changes that are going on in the body, such as the metabolism (the process by which cells change food into energy after food is digested and absorbed into the blood) of the heart muscle. It employs a radioactive drug (tracer) to show this activity.

PET is different from other nuclear medicine examinations in the sense that PET detects metabolism within body tissues while other types of nuclear medicine examinations detect the amount of a radioactive substance collected in body tissue in a certain location to examine the tissue's function.

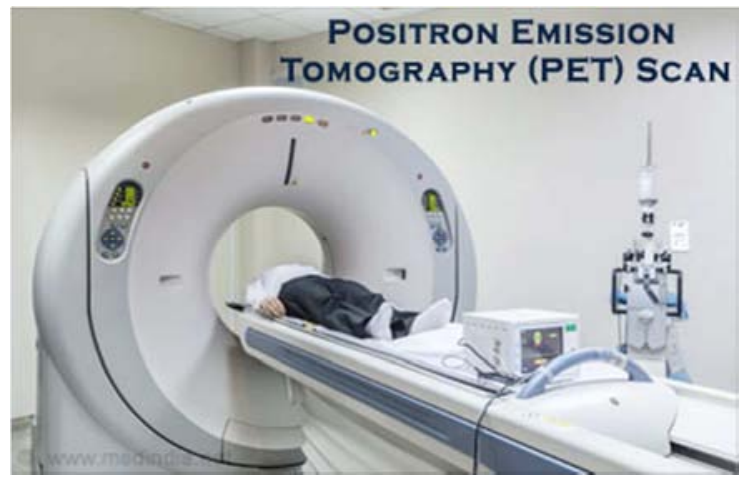

Figure 6. Procedure for Positron Emission Tomography [10].

PET scan is different from CT scan and MRI with the type of images it produces. CT scan and MRI scan reveal the structure of the body, location of the lesion or tumour but PET scan reveals the functions like glucose metabolism, blood supply, oxygen levels, et cetera.

Figure 6 shows a typical Positron Emission Tomography Procedure while Figure 7 depicts the difference between MRI and PET [10]. 


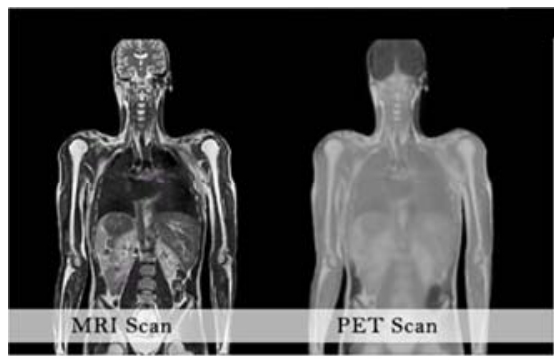

Figure 7. Difference between MRI and PET Scan [10].

\section{vi. Arthrography}

Arthrography sometimes refers to as arthrogram, is a type of imaging test used to look at a joint, such as the shoulder, knee, or hip. It may be done if standard X-rays do not show the needed details of the joint structure and function.

There are some class of imaging in which an X-ray could not show enough detail to pinpoint the problem. For example, when the bone is fractured, cartilage is wearied down or when the ligament is torn. And sometimes, standard imaging, like an X-ray, does not show enough detail to pinpoint the problem. Arthrography is indicated for the following problems:

1. Unexplainable Pain

2. Something feels off in one's joint

3. When the is a problem moving a joint

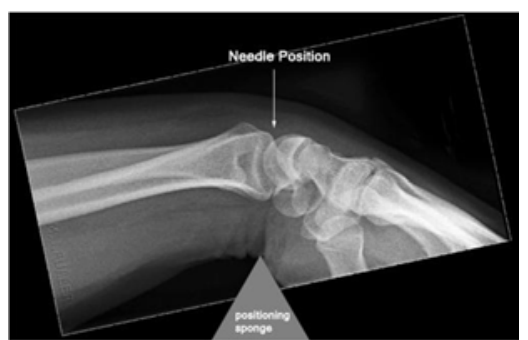

Figure 8. Radiocarpal Joint Technique for needle placement in wrist arthrography of the proximal compartment [11].

Figure 8 shows a Radiocarpal Joint Technique for needle placement in wrist arthrography of the proximal compartment. The wrist is flexed over a positioning sponge in a way that radial articular the surface is profiled.

\subsection{Non-X-rays Based Technologies}

\section{i. Magnetic Resonance Imaging}

Magnetic Resonance Imaging (MRI) is a versatile, noninvasive imaging technique which yields structural

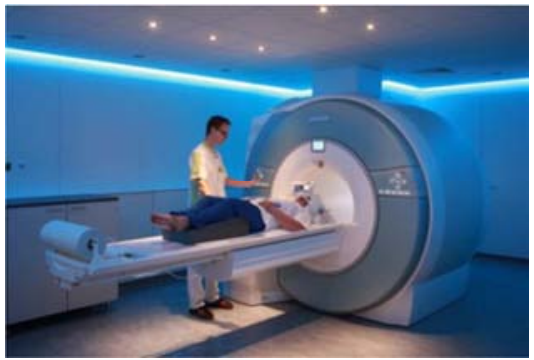

Figure 9. 3T Magnetic Resonance Imaging (3T MRI)
Figure 9 depicts 3T Magnetic Resonance Imaging (3T MRI) in use at Wageningen University \& Research, Shared Research Facilities. It shows anatomical and functional images of the human body parts which use radio waves and magnetic fields in an examination of the organs as well as other structures in the body.

Figure 10 shows the MRI of the brain of a healthy person's brain which is shown on the left-hand side of the figure as well a brain of the patient with Alzheimer's disease by the right.

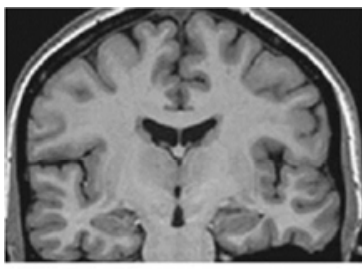

Healthy

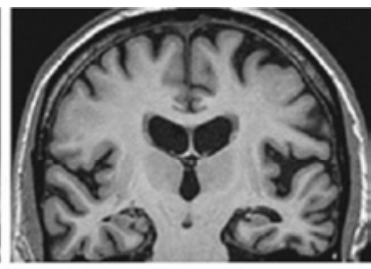

Alzheimer's
Figure 10. Magnetic Resonance Imaging (MRI) sample for a healthy person's brain as well as that of an Alzheimer's patient [12].

\section{ii. Olfactometer}

An olfactometer is an appliance that is used to detect and measure odour dilution. It is used in conjunction with human subjects in laboratory settings to quantify and qualify human olfaction especially to gauge the odour detection threshold of substances. Figure 11 shows the 8-channel olfactometer in use at Wageningen University \& Research, Shared Research Facilities in Wageningen, Netherlands

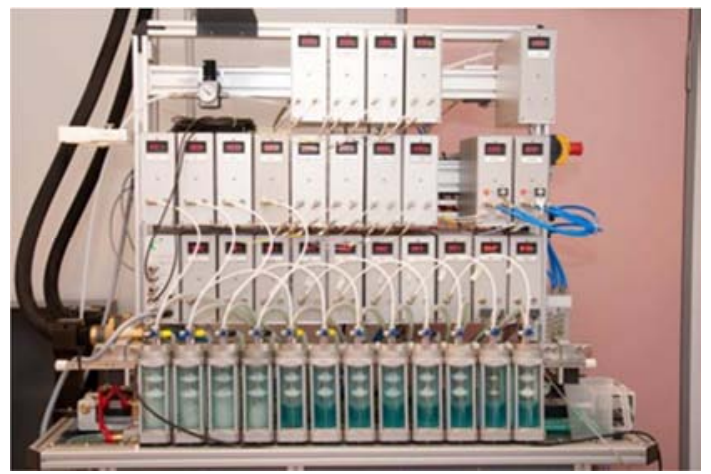

Figure 11. 8-channel olfactometer (courtesy: Wageningen University \& Research, Shared Research Facilities).

An 8-channel olfactometer is used for accurate and controlled administration of odours to a participant. This instrument can be used in combination with 3T MRI for functional MRI studies.

\section{iii. Nuclear Medicine}

Nuclear medicine is a general term that encompasses the use of radioactive materials in medicine. However, when applying it to imaging, it typically refers to the use of radioactive tracers (sometimes called radioactive markers or radiopharmaceuticals). The tracers are radioactive materials that are injected or swallowed so that they can travel through the digestive or circulatory system. The imaging type, in this 
case, is molecular imaging which uses a variety of methods to visualize biological processes taking place in the cells of organisms. Small amounts of radioactive markers, called radiopharmaceuticals, are used for molecular imaging. The radiation produced by the radioactive materials is then detected to create an image of those systems. Figure 12 is a sample radioactive tracer image of human organs.

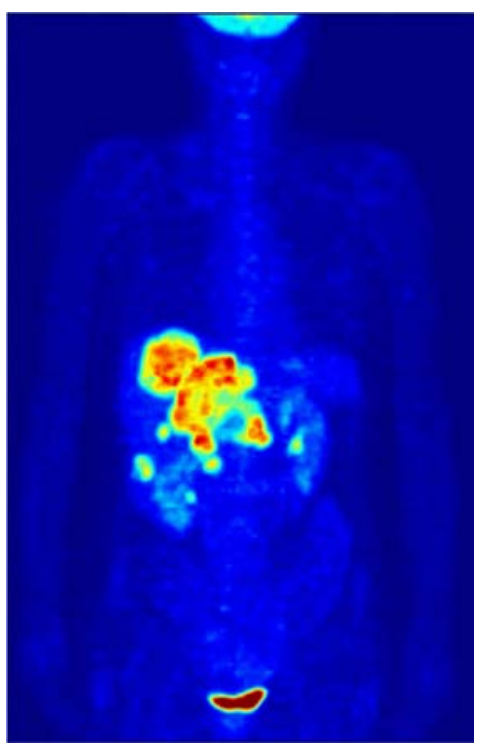

Figure 12. Image of Radioactive tracer [4].

\section{iv. Ultrasound}

Ultrasound uses high-frequency sound waves, which are reflected off tissue to create images of organs, muscles, joints, and other soft tissues thereby producing pictures of the inside of the body. It is typically used to assist in diagnoses of the primary causes of pain, swelling and infection in the internal organs of a body. It is also used to examine a baby in pregnant women, brain and hips in infants, help to guide biopsies, diagnose heart conditions, and assess damage after a heart attack. Ultrasound is safe and noninvasive. Also, it does not use ionizing radiation. A typical ultrasound imaging is shown in Figure 13.

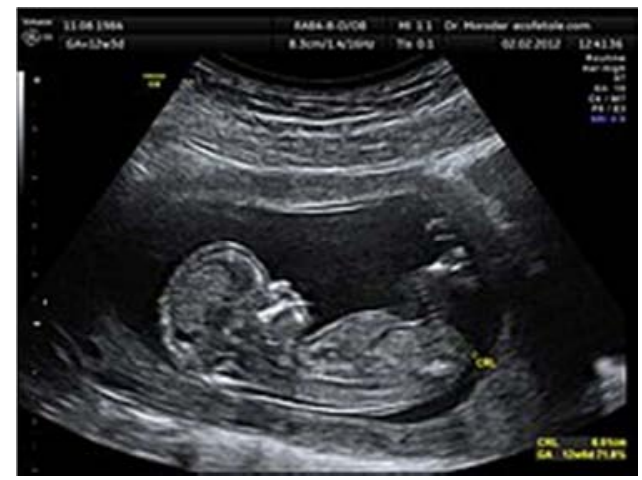

Figure 13. A 12 weeks of pregnancy Ultrasound image (sonogram) of a fetus in the womb (bidimensional scan) [13].

\section{v. Scintigraphy}

National Cancer Institute describes scintigraphy otherwise known as Radionuclide Bone Scan as a procedure that produces pictures (scans) of structures inside the body, including areas where there are cancer cells [14]. The procedure is employed in diagnosing, staging, and monitoring of disease. In this case, a small amount of a radioactive chemical (radionuclide) is injected into a vein or swallowed. A machine equipped with a special camera moves over the person lying on a table and detects the type of radiation given off by the radionuclides. An image of the areas the radionuclide is built-up is displayed on the computer monitor.

A typical sample of the outcome of bone scintigraphy culled from the research in the UC Davis School of Veterinary Medicine) is shown in Figure 14.
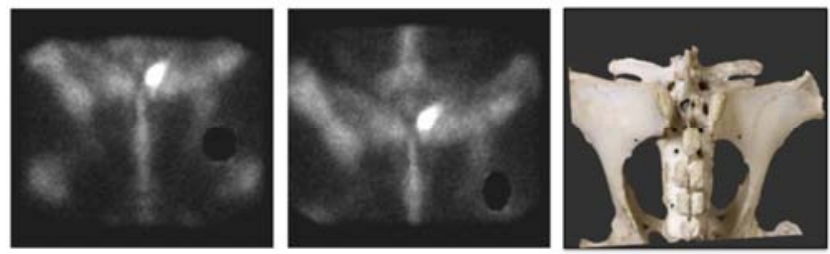

Figure 14. A scintigraphy Result Sample (Source: UC Davis School of Veterinary Medicine).

These are images of the pelvis with the camera above the horse so that the lumbar spine is at the top of each image and the wings of the ilium extend to either side. There is an asymmetry in the pelvis of this horse with severe focal radiopharmaceutical uptake of the right tuber sacrale or point of the croup. This represents a fracture in this patient.

\section{vi. Dual Energy X-ray Absorptiometry}

Dual-energy X-ray absorptiometry (DXA or DEXA) is a test that uses low-dose $\mathrm{X}$-rays to measure bone mineral density, including calcium content, in a section of bone.

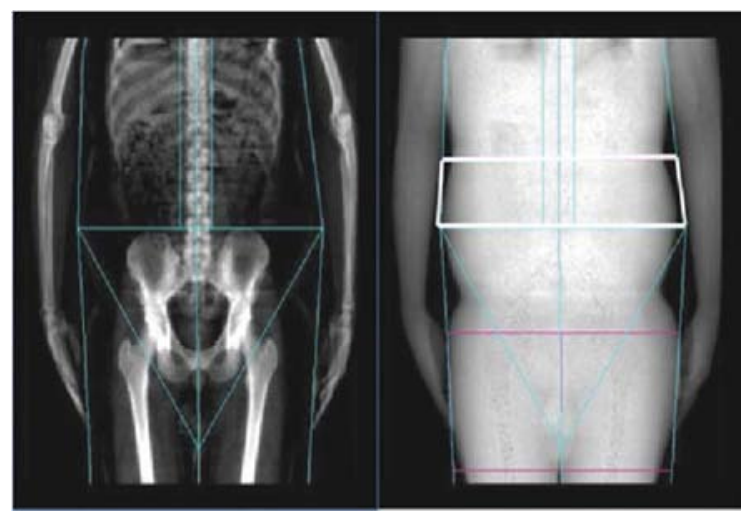

Figure 15. Dual-energy X-ray absorptiometry [15].

Thus, it is used to determine the density of the bone to assess its strength. The medical condition termed osteoporosis is diagnosed by DXA. It is used in combination with risk factors (the so-called "FRAX" method. Also, the estimation of bone fracture risk can be determined by DXA.DXA screening is used to uncover bone problems of the skeleton, for example in people who have unexplained back pain or who have experienced a loss in height of more 
than an inch in a year.

Clinical Indication which is generally related to the conditions concerning poor bone health, according to the Department of Radiology and Bio-medical Imaging gives the category of people who should consider assessment with DXA is as follows:

1 Women 65 and older and men over age 70 assessment.

2 Women under age 65 and men ages 50 - 70 who have risk factors such as:

3 a fracture over age 50

4 rheumatoid arthritis or chronic kidney disease

5 eating disorders

6 early menopause (from natural causes or surgery)

7 history of hormone treatment for prostate or breast cancer

8 a significant loss in height

9 smoking

10 family history of osteoporosis

11 taking corticosteroids (prednisone, methylprednisolone) every day for 3 months or more

12 three or more alcoholic drinks per day on most days. [16]

DEXA scan is described as been helpful in various conditions such as:

1 to assess how well the osteoporosis treatment is working.

2 if one is suffering from back pain which may be caused due to spine fractures.

3 if one has lost height of half-inch or more within one year.

4 women who have reached menopause.

5 people with a personal or medical history of hip fracture.

6 smokers.

7 post-menopausal women who are tall or thin

8 men with clinical conditions like rheumatoid arthritis or chronic kidney disease

9 people with a thyroid condition

10 people with Type 1 diabetes, liver disease, kidney disease

11 people who have had a fracture after mild trauma. [17]

Figure 15 depicts a typical sample of dual-energy X-ray absorptiometry (DXA) bone and tissue images. The straight lines defined by the skeletal image in the left of the figure.

\section{vii. CT-SCANNER}

Lawrence described CT, or CAT Scans (sometimes refers to as computerized axial tomography) as special X-ray tests that produce cross-sectional images of the body using X-rays and a computer [18]. CT scans are also referred to as computerized axial tomography. CT Scan can be done quickly and it is a very essential tool in assisting a doctor to make a diagnosis. CT can spot small tumours in the body which could not be seen by the conventional x-ray. For example, the CT scan can help doctors to visualize small nodules or tumours, which they cannot see with a plain film $\mathrm{X}$-ray. Figure 16 denotes a CT scan of the neck.

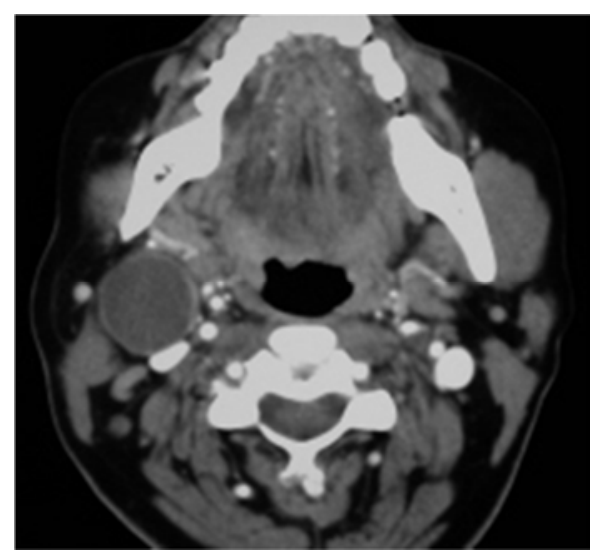

Figure 16. Neck CT scan [18].

\section{Uses and Benefits of Medical Imaging}

The techniques in medical imaging could be used in different capacities and circumstances. For instance, radiography is used whenever we want the images of bone structures to look for breakages. MRI scanners are typically used to take images of the internal tissues and sometimes brain. Nuclear medicine is used whenever the inside of the digestive or circulatory systems, such as to look for blockages, is needed. Ultrasound can be used to picture foetus in the womb especially when high resolution is not necessary. The development of "small magnet" would eventually lead to portable Magnetic Resonance Imaging (MRI) in addition to presenting less anxiety in patients with claustrophobia symptom [19]. The new scanners in operation today are less restrictive and can accommodate individuals with ease. A vast amount of image materials could be rapidly analyzed and classified to provide a reliable, timely and accurate diagnostic for the use in medical health care delivery system.

Also, cloud computing advancement offers incredible opportunities to process a vast volume of data and arrive at a timely and correct medical diagnosis. These operational and clinical data could be made available through cloud computing. In this case, big data Analytics and advancement in Data mining usher a very great advantage when dealing with a medical image.

The 3D technologies in diagnostic radiology have the capabilities to create high-quality images thereby greatly increasing the diagnostic potentials. It should be noted that traditional ultrasound technology produces low-resolution images and sometimes requires great efforts to better result. But 3D technology offers the opportunity of producing better CT scan resolution and improves patient health care delivery system. Besides, Digital Mammography, also called FullField Digital Mammography (FFDM) is defined as a mammography system in which the X-ray film is replaced by solid-state detectors that convert X-rays into electrical signals [20]. For example, the images of the breast are produced by the electric signals and can be viewed using a computer screen. Digital Mammograms are better than traditional ones and specifically best suited for women with thick breast 
tissues, women nearing the menopausal age, premenopausal women, and those below 50 years. Stanford Health Care and Devin describe a digital breast tomosynthesis (DBT) as consisting of a series of images which are generated along with the breast and make it possible for radiologists to view each tissue independently thus improving the lesion visibility and make it possible for earlier detection of cancer [19, 20]. It produces a lower error rate. For instance, in Computeraided detection (CAD) systems, a digitized mammographic image that can be obtained from either a conventional film mammogram or a digitally acquired mammogram is used. The algorithm designed to locate the cancer searches for abnormal areas of density, mass, or calcification in the breast which may provide a clue to the presence of cancer. The CAD system indicates these spotted areas on the images.

Rede was recently released by Konica Minolta Healthcare in the United States [21]. It is a medical image management solution for specialist clinics. Rede PACS and Rede Mini PACS are designed for specialist practices, such as orthopaedic, urgent care and family medicine clinics to give features and tools necessary to optimize and simplify imaging workflow. It provides fast access to images from any device scaled for the specialist care market

Furthermore, Artificial Intelligence has played a major role in exploiting growth in this knowledge area to benefic medical diagnostic and delivery system. For example, Artificial Intelligence can categorize cancer risk of lung nodules. According to the Imaging Technology News, computed tomography (CT) scans for people at risk for lung cancer led to earlier diagnoses and improve survival rates [22]. The potential clinical utility of this deep learning algorithm to revise the probability of cancer among IPNs with the potential to decrease invasive procedures and shorten time to diagnosis. The study published in the American Journal of Respiratory and Critical Care Medicine shows that an Artificial Intelligence strategy can correctly assess and categorize these indeterminate pulmonary nodules (IPNs). The algorithm developed by the team of researchers in a very large dataset $(15,693$ nodules) reclassified IPNs into low-risk or high-risk categories in over a third of cancers and benign. This is a significant improvement over the present conventional risk models clinicians.

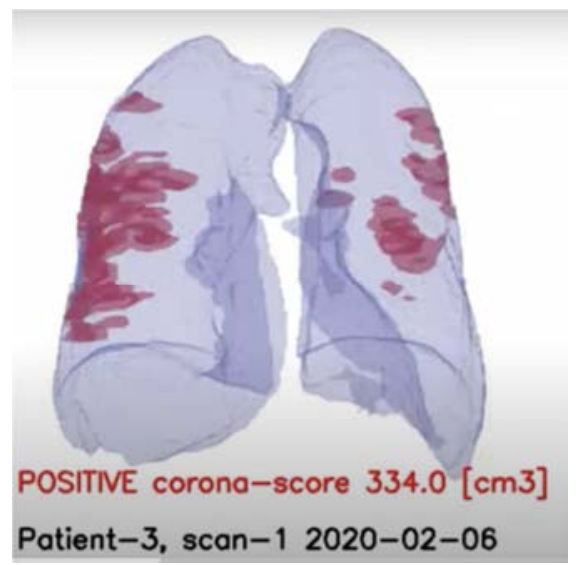

Figure 17. A Sample of COVID-19 Patients Chest Imaging [21].
Besides, the Imaging Technology News reported RADLogics deployments and installation of the company's AI-powered solution to support chest computed tomography (CT) imaging for COVID-19 (Coronavirus) patients at hospitals and healthcare providers in China, Russia, Italy, Serbia and Brazil, and other parts of the part world [23]. Figure 17 shows the chest imaging for COVID-19 Patients by enrolling the use of Artificial Intelligence.

Moreover, it would be helpful to briefly highlight more equipment use in Nigeria for generating Medical Images. In this category are some few types of equipment from LISTEM, one of the leaders in Digital Imaging System. Among the endless list are one high-frequency Radiography and fluoroscopy X-ray TV system (REXFT System), Highfrequency radiography system (REX_R System), General Radiography X-ray system (DXG-SYSTEM). Apart from these areas of the generator, in other areas of mobile signal $\mathrm{C}$-Arm, we have high-frequency mobile C-arm X-Ray TV system (CSM_20HF), SM_25HF. Their specialist product includes high-frequency radiography X-Ray system (CST21HFII), Ext purpose Radiography X-Ray, radiography Xray system X-ENT II. Under tube stand and table there exist the floor to ceiling tube stand (SFC-31) et cetera. under the category of Table, we have six-way floating Top bucky table $($ KOB-60N) $+90 /-15$ table with spot film device. Other products from this company include X-Ray Beam collimator, high-frequency mobile X-Ray units, veterinary X-Ray system et cetera.

The president of Sanger Heart and Vascular Institute with Atrium Health, in Charlotte, North Carolina, and a board member with the American Society of Echocardiography (ASE), when discussing the impact of COVID-19 (SARSCoV-2) on the cardiovascular service line and cardiac imaging said the virus has led to use of computed tomography (CT) in the frontline cardiovascular imaging modality to evaluate chest pain and for COVID-19 pneumonia imaging [24].

\section{Process in Generating, Capturing, Storage and Reporting in Digitized Imaging}

A digitized imaging system is often distributed into the processes of producing the image. Typical of the processes are image generation, capturing, storage, retrieval, transmission, display, analysis, visualization and reporting. The generation of the image could be defined by the energy source used. Such energy sources include X-rays, ultrasound, MRL, radiofrequency and radioactive decay. Other developments are rapidly springing up from research. These include inverted X-Ray, functional MRL and 3D ultrasound. The modality for generating digitized imaging could be split into two groups: ionizing radiation-based modalities such as X-ray, CT (ORLAT) and nuclear medicine and non-ionizing modalities such as ultrasound and MRI. It should be noted that a significant portion of the medical imaging market 
traditionally available in Nigeria is X-Ray. This may be attributed to the high-quality imaging resolution of X-ray based technique. However, other digitized imaging technique such as fluoroscopy, endoscope, EEG signals (not formally an image) dental X-rays, pathology sections (visual lightbased images) also generate a similar set of data that is similar to those generated by medical images. The capture of digitalized medical images involves storing it in film or videotape. The film is viewed by a radiologist, interpreted and stored in the library. The predominant method of capturing film on tape has recently given way to digital capture of images and digitized form of storage. This is the future and has a lot of distinct advantage. The adoption of this method would lead to a reduction in cost.

The storage and retrieval technique for digital image depending on the type of storage required. The storage may be needed to achieve a short time or long-time requirement. The short time requirement is achieved using local server employing RAID (Redundant Array of Independent Disks) technology. RAID is a method used to standardize and categorize fault-tolerant disk systems. Through the method, we can hold image waiting to be retrieved as well as using it as a buffer in case of network failure. The long-time need is met by using centralized storage system with CD, DVD and tape jukeboxes and lately Cloud-based storage and retrieval, External Disk, Flash drives.

Besides, despite the obvious advantages image storage and retrieval can also have the following challenges in the following areas:

1. Data storage and database health monitoring

2. Security: user account management, access control administration.

3. Migration/lifecycle rule sets: monitoring, editing, administration.

4. Network and storage device health and performance.

5. Planning tools for upgrade and expansion. [25]

However, the advent of the open system is paving way for web browser technology which is easing some of these challenges. Images are centrally stored and accessed via a web browser. Another way images in medical imaging system could be stored is through compression. The compression minimizes the storage sizes and transmission of digitized images. There are two different types of compression vis-à-vis: lossless compression and loss compression. The former retains all the original data in the compressed version whereas the latter drops data for the sake of further reducing the file size. The degree of loss here varies depending on imaging application [26].

Picture archiving and communication system (PACS), a network system, was designed to transmit store and retrieve digital medical images. It is more or less a 'virtual film library' backend to radiologist's report. Digital Images Communication in Medicine (DICOM) was designed as a standard file structure so that digitized imaging from the different company could have the same structure and be able to integrate. There is a various range of transmission depending on size and application. Increase bandwidth in an application such as real-time viewing is normally characterized by an increase in cost. Canada is particularly paying good attention to research in the area of developing telecommunications infrastructure and a couple of companies are championing the improve telecommunication (in term of supporting hardware and software).

Display of Digital images record can be achieved in two ways. One of the ways is by producing a hard copy of images in the form of prints. Another is producing the softcopy for viewing on the computer. The film can be illuminated by light for direct viewing too. DICOM is working hard to see that image produce are of high quality irrespective of terminals used to view the image. For example, TrueGre ${ }^{\circledR}$ monitors are treading this path.

Furthermore, the analysis, visualization and reporting of the digitized image would be considered before going to the next session. The traditional way of analyzing in the filmbased environment is for the radiologist to physically examine the film and make the necessary recommendation to the physician. This crude way has given way to the computer-based expert system which automates the process of examining and eliminating the deformation [27]. Visualization has to do with integrating the information and rendering it into the proper form such as, for example, a 3D object which allows for better presentation and interpretation of the information. The traditional way of reporting is for the radiologist to dictate the report for typing, which is reviewed, corrected and approved by the radiologist. The report is then stored in the radiological information system (RIS). Research is in progress to develop a fully efficient speech transcription device to convert the radiologist report directly to speech to text report. The prototype machine is already in use and the ultimate goal is to produce a full, reliable speech recognition, which is expected to be in place in the nearest future. Also, work is going on toward developing standard reporting formats [28]. Integration of imaging modalities with archival systems (PACS) is perhaps the biggest opportunity and challenges for medical images. Two of the clinical changes have been described as one; the distributed and divergent nature of health care information (i.e. it enters in form multiple points in multiple formats) and two, the incompleteness of existing information interchange standards. Also, two standards in prominent use in healthcare-related information systems were given as DICOM for imaging equipment and HL 7 (Health Level 7) for exchanging text base information systems, patient registration, admission, lab results, discharge information between hospital information systems (HIS) and Radiological Information Systems (RIS). DICON provides uniformity and compatibility between medical imaging equipment while HL 7 provides the same between RIS to HIS. It is also worthy to note that Interface engine otherwise known as "Black Boxes" are available which also provide connection of non-DICOM 3.0 legacy equipment to a DICOM 3.0 compliant network. Mitra imaging Inc. of Waterloo PACS broker system is widely used by PACS vendors in interfacing between PACS modalities and the RIS [29]. 


\section{Prospects of Medical Imaging in Nigeria}

The expected contribution of medical imaging in Nigeria could be enormous. The use of medical imaging could lead to reduced cost and improve efficiency. It would also lead to reducing medical error associated with traditional medical therapy. In the United State of America, Canada, Sweden and host of other advance countries where medical imaging technology has taken root, it has been found that it makes a substantial impact on medical quality and safety by integrating relevant automated decision making and knowledge acquisition tools into the practices of medical therapies. The use of imaging technology has been promoted as having tremendous promise in improving the efficiency, cost-effectiveness, quality and safety of medical care delivery in Canada and US.

Given insight into the occurrences of medical error in medical practice in Nigeria, reducing the effects of such errors in hospitals lies in effective implementation of medical imaging technology in the health care delivery system. Medical Imaging could be an important tool in reducing the rate of medication errors that occur in the care of infants and children. It would then make a substantial impact in medical quality and safety by integrating relevant automated decision making and knowledge acquisition tools into the practice of medical providers, thereby reducing errors of omission that result from gaps in provider knowledge or the failure to synthesize and apply that knowledge in clinical practice with the system when integrating with larger HIT systems. It has been found to improve decision making and appropriate use in therapeutic agents. Medical imaging technology offers the opportunity to monitor and improve clinical quality, improving information access and reducing duplication. The use of ambulatory Electronic Health Records (EHRs) equipped with imaging technology offers the opportunity to monitor and improve clinical quality, improving information access and reducing duplication. In ambulatory health care environments, the use of medical imaging can improve the efficiency and financial health of the practice.

Furthermore, virtual reality in medical imaging practice in Nigeria with emphasis on imaging diagnostic and therapy can lead to improving surgical results. An example that readily comes to mind is the use of Laparoscopic Simulators for training development of an application that simulates human response to medication. The training can be practised using a virtual environment without reducing the supply of non-renewable resources. Also, judging by the crisis in the energy sector in Nigeria, adopting a reliable medical delivery system that prioritizes imaging diagnostic and therapy would lead to nonrenewable resource savings. For example, the use of simulator saves precious resources such as cadavers and animals. If medical practitioners train using simulators, the demand for nonrenewable resources can be drastically reduced. The training can be practised using a virtual environment without reducing the supply of non-renewable resources.

The use of digitalized medical imaging system can lead to an online diagnosis, remote patient monitoring and compensate for the shortage of medical practitioners. This is particularly the case in telemedicine. Nigeria is a country with the majority of the population living in remote areas and with the current trend in brain drain occasioned by a bad economy, telemedicine would open the way for the population in a rural setting and opportunity to benefit from medical services and reduce the mortality rate in Nigeria.

Good medical in an imaging system is arguably an effective pill for good disease management. It has the potential to improve productivity. Another prospect in Nigeria would be the avoidance of morbidity because of improves patient diagnostic.

\section{Challenges of Medical Imaging in Nigeria}

One of the most serious challenges faced by Nigeria is the high costs associated with the acquisition and implementation of medical imaging in Nigeria. The costs could be categorized into two parts. The cost associated with the system itself. This covers the hardware, software license, maintenance and support. The second part is the implementation cost (training, temporarily loss of productivity et cetera) for a country that is still struggling with the challenge posed by unemployment, illiteracy, economic recession and other socio-economic woes occasioned by the crisis in the Niger Delta, Boko Haram insurgency, epidemic disease (e.g. Polio, COVID19 Pandemic et cetera) and brain drain. Also, having enough capital and necessary foresight to implement medical imaging system could be a daunting challenge. Even in advance country financing, such a project is quite challenging. One study estimated the cost for ambulatory HER to be $\$ 9,700$ per provider - $\$ 1,600$ for annual software license and $\$ 1,800$ for support and main license [30]. In Sweden, the cost of developing a primary health care setting of 50 staff was estimated at $\$ 2400$ in the first year. In a large HMO with 13 outpatients care location in Ohio, the use of a homegrown ambulatory HER was estimated to cost $\$ 10 \mathrm{M}$ with the additional expense of $\$ 630,00$ for printing networks and 110 allied professionals. The second cost would be the cost of the implementation process. Among various studies conducted, the Sweden study used a socialite perspective and included the cost of training and unexpected cost of selftraining during' working hour. Loss of normal activities in leisure was also found to increase in administrative workload extra service, and medical records summary. The cost was estimated at $\$ 75,000$. However, studies showed an annual saving of $\$ 3,700,000$ associated with improving efficiency resulting from reduced medical record room and support staff, elimination of clinical form and automatic collection of billing data. In a country like Nigeria where there is high poverty among the majority of her citizens, high unemployment rate and a large number of illiteracies, implementing medical imaging is very tasking. High initial cost, non-quick returns on investment may discourage the use 
of imaging technology in the administration of the health delivery system. At present, most virtual reality applications are based on a Reduced Instruction Set Computer (RISC) platform whose cost is beyond the reach of an average therapist and medical practitioners. Another barrier would be access to internal training and insufficient computer operational skill.

An additional barrier is insufficient research about Information Technology (IT) in paediatrics. Most of the physicians lack sufficient computer knowledge to leverage the improved use of IT in their professional practices and may need government policies to shift to sensitize them. One cannot but acknowledges the school of thought who feels apprehensive by the introduction of IT as suggested by Falaki [31]. These groups of people maintain a sustained opposition to any technological change and pose a great challenge to quick acceptance of new ways of doing things.

The infrastructural decay as a result of the military intervention in politics and corruption by unscrupulous politicians in Nigeria remains a major challenge. There is hardly a town or city in Nigeria where there is an adequate supply of electricity. Hence, integrating imaging technologies into the medical delivery system becomes a difficult task. Also, lack of reference standard associated with all the existing application in the health sector can be considered "one-off" creation tied to proprietary hardware and software, which has to be optimally turned by process of trial and error. This makes it more difficult to adapt for use other than those in which they were specifically developed. The lack of good organizational structure in most of the national hospital and medical facilities is a major problem. Good implementation of imaging system requires tiding organizational structure, which is adaptable to changes in the need of the organization. Typically, the software should be adaptable to the need of the organization. The organization must be amenable to developing new policies, procedures and training of staff.

Medical imaging System (MIS) is believed to be a vital component in the quest to improve medication safety in paediatrics. These special requirements, combined with a small commercial market for pediatric MIS systems relative to the adult population, implement in the pediatric is setting challenging and costly. Almost all the essentials are at present not available in Nigeria. It, therefore, becomes a practical challenge to formulate well research policies to put these in place for the eventual realization of DMIS in the country.

The government needs to change its look-warm attitude to the issue of introducing IT to the medical field. Quite an appreciable percentage of our medical personnel lack basic knowledge of IT. It should be noted that implementing DMIS without the active participation of these experts would be a failure. The current trend in the world is the integration of IT into virtually every field including medical sphere, Nigeria could not be an exception. People who are well bread and who are good technocrat should be appointed to the key position for the process to succeed.

Power sector in Nigeria should be urgently liberalized and privatized to pave way for the feasible implementation of
DIS. The process of economic re-engineering already started in Nigeria should be relentlessly pursued to eliminate poverty in the land. After all, the axiom that a hungry mind is an angry mind is true. The hungry mind cannot implement an imaging system; unemployment problems in the Nation should be tackled. The Nigeria National Orientation Agency (NOA) should be mobilized to sensitize the populace to the importance of MIS. Moreover, there should be a policy framework in favour of MIS in Nigeria medical practices.

Finally, the introduction of MIS in Nigeria offers a rare opportunity to revive the ailing medical sector which is in a state of comatose. Introduction of DMIS in Nigeria, the most populous state in Africa, would have an impact on the other Africa sub-regions. If DMIS succeeds in Nigeria there would be an improvement in all areas of medical practices. Hence infant mortality rate would be reduced; there would be a reduction in cost and quick delivery of medical services would be achieved.

\section{Conclusion}

The present state of medical health care delivery system in Nigeria is not desirable. The benefits accruing from integrating medical imaging into the system is eluding Nigerians. Despite the enormous opportunities, the medical imaging could provide for the health care delivery system, the stakeholders are not showing enough concern, enthusiasm or commitment needed to advocate and mobilize the health practitioners for improving Medicare using a medical imaging system. The system is bedevilled by multiple challenges just like other facets of the nation socio-political and economic systems. Nigeria health care delivery system would be transformed by introducing and integrating medical imaging into the system. Apart from the immense economic opportunities, it would provide, the citizens would also benefit from improved health services and thus invariably increase the wealth of the nation. After all, the common saying "a healthy nation is a wealthy nation" has been found applicable to all nations in all generations.

\section{References}

[1] World Population Review (2019) Nigeria Population 2019 [online] Available at: http://worldpopulationreview.com/count ries/nigeria-population/ (Accessed: 19 July 2019).

[2] Adeola, O. S., Alese, B. K. and Falaki S. O. (2007) National Crime Intelligent System. Information Technology Journal, 6 (5): 633-647.

[3] Ojoma Akor and Olayemi John-Mensah (2018,) Nigeria: 'How Nigeria Can Utilize Radiography to Improve Healthcare Delivery Daily Trust, June 19 (Abuja).

[4] David Wood (no date) Medical Imaging Techniques: Types \& Uses. Chapter 7, Lesson 7", GACE Prep First Lesson GACE Science (524): Practice \& Study Guide [online] Available at https://study.com/academy/lesson/medical-imagingtechniques-types-uses.html (Accessed: 01 June 2020). 
[5] UrologyAustin (2010) KUB Radiography [online] Available at: https://urologyaustin.com/urology-services/kub-radiography/ (Accessed: 01 June 2020).

[6] Radiology.org (2020) Intravenous Pyelogram (IVP) [online] Available https://www.radiologyinfo.org/en/info.cfm?pg=ivp (Accessed 20 May 2020).

[7] Rathi, V., Agrawal, S., Bhatt, S., \& Sharma, N. (2015) Ureteral Dilatation with No Apparent Cause on Intravenous Urography: Normal or Abnormal? A Pilot Study Advances in Urology, 2015, 1-6. DOI: 10.1155/2015/681836.

[8] Chicilo F, Hanson A. L, Geisler F. H, Belev G, Edgar A, Ramaswami K. O, Chapman D and Kasap S. O (2020) Dose profiles and $\mathrm{x}$-ray energy optimization for microbeam radiation therapy by high-dose, high-resolution dosimetry using Sm-doped fluoroaluminate glass Figures and Monte Carlo transport simulation. Physics in Medicine \& Biology, 65 (7).

[9] U.S. Food and Drug Administration (2020). Fluoroscopy [online] Available at: https://ww w.fda.gov/radiation-emittingproducts/medical-x-ray-imaging/fluoroscopy (Accessed: 22 May 2020).

[10] Himabindu Venkatakrishnan (2020) What is Positron Emission Tomography (PET) Scan? Medidia Health $\begin{array}{llll}\text { Information } & \text { Anline] ailable }\end{array}$ https://www.medindia.net/patients/patientinfo/positronemission-tomography-scan.htm (Accessed: 25 May 2020).

[11] WikiRadiography (2020) Wrist Arthrography [online] http://www.wikiradiography.net/page/Wrist+Arthrography (Accessed: 25 May 2020).

[12] Juan Manuel Fern'andez Montenegro (2018) Alzheimer's Disease Diagnosis Based on Cognitive Methods in Virtual Environments and Emotions Analysis. A thesis submitted in partial fulfilment of the requirements of Kingston University for the Degree of Doctor of Philosophy (PhD).

[13] Wikipedia (no date) The free encyclopedia [online] Available at: https://en.wikipedia.org/wiki/Ultrasound (Accessed: 01 June 2020).

[14] National Cancer Institute (2019) NCI Dictionary of Cancer Terms. NCI Dictionary of Cancer Terms. September 5.

[15] Kaul, S., Rothney, M., Peters, D. M., Wacker, W. K., Davis, C. E., Shapiro, M. D., \& Ergun, D. L. (2012) Dual-Energy X-Ray Absorptiometry for Quantification of Visceral Fat. Obesity. Silver Spring, Md., 20, 1313 - 1318.

[16] Department of Radiology and Bio-medical Imaging (no date) Bone Density Scan (DXA or DEXA). University of California, San Francisco.

[17] Hospital (Feb 28, 2019) Amandeep Hospital Introduces Dualenergy X-ray Absorptiometry (DEXA) Scan in Amritsar [online] Available at https://medium.com/@amandeephospital digitalasr/amandeep-hospital-introduces-dual-energy-x-ray-ab sorptiometry-dexa-scan-in-amritsar-bf95d6edb3bc (Accessed: 02 June 2020).
[18] Lawrence M. Davis (2019) CT Scan (CAT Scan, Computerized Axial Tomography. Emedicinehealth [online] Available at: https://www.emedicinehealth.com/ct_scan/article_em.htmLoui se

[19] Devin Morrissey (2018). Advancements in Medical Imaging Technology [online] Available at: https://www.ecnmag.com/ blog/2018/09/advancements-medical-imaging-technology (Accessed: 02 April 2019).

[20] Stanford Health Care (n. d.), Full-Field Digital Mammography (FFDM) [online] Available at https://stanfordhealthcare.org/ medical-tests $/ \mathrm{m} /$ mammogram/digital-mammography.html (Accessed: 20 July 2019).

[21] Imaging Technology News (April 28, 2020) Konica Minolta Releases PACS Solutions for Specialty Clinics. Available at: https://www.itnonline.com/content/konica-minolta-releasespacs-solutions-specialty-clinics (Accessed 24 January 2021).

[22] Imaging Technology News (April 27, 2020) Artificial Intelligence Can Categorize Cancer Risk of Lung Nodules. [online] Available at: https://www.itnonline.com/content/artifi cial-intelligence-can-categorize-cancer-risk-lung-nodules (Accessed on 24 January 2020).

[23] Imaging Technology News (April 21, 2020) RADLogics Expands Deployment of AI-Powered Solution to Support Chest Imaging for COVID-19 Patients [online]. Available at https://www.itnonline.com/content/radlogics-expandsdeployment-ai-powered-solution-support-chest-imagingcovid-19-patients (Accessed on 24 January 2020).

[24] Geofry Rose (n.d.) VIDEO: CT and POCUS Emerge as Frontline Imaging Modalities in COVID-19 Era. Imaging Technology news [online] Available at: https://www.itnonl ine.com/videos/video-ct-and-pocus-emerge-frontline-imagingmodalities-covid-19-era (Accessed: 19 April 2020).

[25] Robert Glickman (2020) Data Storage Management. Archiving: Fundamental of Storage Technology, Society for Imaging Informatics in Medicine, Leesburg, VA 20176.

[26] Bulpitt, C. J., Beilint I. J and Coles E. C., (1976) Randomized control trial of computer held medical record in hypersensitive patients. Br. Med; 1 (6011): 677-9.

[27] Furan, D. J., Chen, W., \& Yang, L. (2011) Automated image interpretation computer-assisted diagnostics. Analytical cellular pathology (Amsterdam), 34 (6), 279-300.

[28] Caroll, A. E., Hornoch, P. and O'reilly E. (2004). The effect of point of care personal digital assistant use on resident documentation discrepancies. Pediatrics; 113 (3Pt 1): 4504.

[29] Brown, B. G., Evans A. and Wall T. (1985). Effect of computer use in the consultation on the delivery of case. $\mathrm{Br}$ Med J. (Clin Res Ed): 292 (6496): 634.42.

[30] Burrack, R. C, and Gimotty, PA. (1997) Promoting Screening mammography in inner city settings. The sustained effectiveness of computerized reminder in randomized controlled trial. Medcare 1997: 35 (9): 921-31.

[31] Falaki S. O, (1984). Microcomputer use in Agriculture Ekiti Akoko Agricultural Development Project (EAADP). 